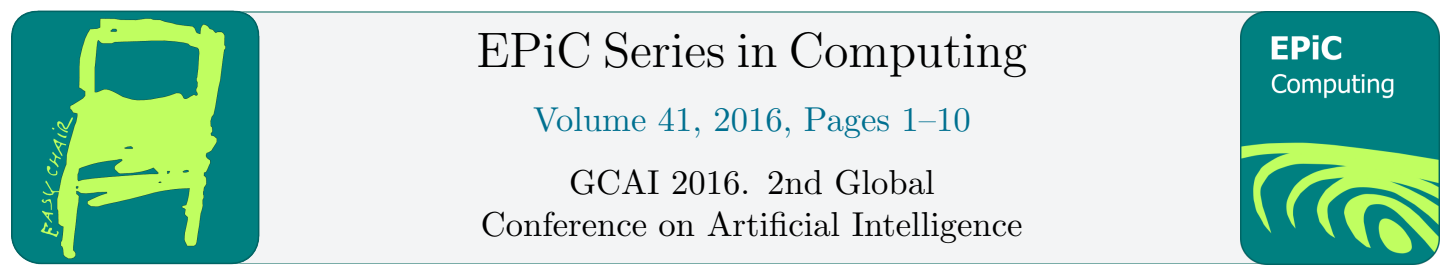

\title{
Tutorial on Reasoning in Expressive Non-Classical Logics with Isabelle/HOL
}

\author{
Alexander Steen ${ }^{1}$, Max Wisniewski ${ }^{1}$, and Christoph Benzmüller ${ }^{1}$ \\ Freie Universität Berlin, Berlin, Germany \\ a.steen $\mid$ m.wisniewski|c.benzmueller@fu-berlin.de
}

\begin{abstract}
Non-classical logics (such as modal logics, description logics, conditional logics, multi-valued logics, hybrid logics, etc.) have many applications in artificial intelligence. In this tutorial, we will demonstrate a generic approach to automate propositional and quantified variants of non-classical logics using theorem proving systems for classical higher-order logic. Our particular focus will be on quantified multimodal logics. We will present and discuss a semantical embedding of quantified multimodal logics into classical higher-order logic, which enables the encoding and automated analysis of non-trivial modal logic problems in the Isabelle/HOL proof assistant. Additionally, TPTP compliant automated theorem proving systems can be called from within Isabelle's Sledgehammer tool for automating reasoning tasks. In the tutorial, we will apply this approach to exemplarily solve a prominent puzzle from the AI literature: the well-known wise men.
\end{abstract}

\section{Introduction}

Computer-assisted reasoning in non-classical logics is of increasing interest in artificial intelligence (AI), computer science, mathematics and philosophy. Several powerful automated and interactive theorem proving systems have been developed over the past decades. However, with a few exceptions, most of the available systems focus on classical logics only. In particular for quantified non-classical logics there are only very few systems available to date.

This situation is in contrast to the relevance of quantified non-classical logics for a wide range of AI application areas such as knowledge representation, multi-agent systems, reasoning with uncertainty, counterfactual reasoning, social choice, etc. Prominent logics for these application areas include, for example, modal logics and hybrid logics [19]), description logics [5]), manyvalued logics [23]) and conditional logics [21, 28]. However, the development of calculi and reasoning systems for these and other non-classical logics is mainly focusing on propositional resp. decidable fragments. Another aspect is that non-classical logics often come with various parameters that need be adjusted for particular application domains (e.g. different combinations of axioms in modal logics, or different conditions such as varying domains vs. constant domains). However, the required flexibility for such parametric logics is not easy to achieve in practical implementations.

Orthogonal to the development of specialized provers, a semantical embedding approach (cf. $[9,12,14,29])$ allows for a quick adaptation of existing higher-order reasoning systems to a 
broad variety of expressive, non-classical logics. Recent related work has focused on automation of quantified conditional logics [6], quantified hybrid logics [31], free logics [14] and many-valued logics [29]. There is empirical evidence that such embeddings can be employed together with reasoning systems for higher-order logic to successfully verify or refute non-trivial arguments in e.g. metaphysics and that they even can contribute new knowledge [18, 16, 8, 15].

In this tutorial we will focus on the application of the embedding approach to modal logics $[12,17]$. More precisely, we will present and discuss some prominent example problems that can be modeled using modal logics. In this paper, we will use the wise men puzzle as a representative example for the approach. The experiments are conducted using the Isabelle/HOL proof assistant [27] and can be run on any computer.

\section{Classical Higher-Order Logic}

The target logic for the presented embedding as well as the logical foundation of the higher-order reasoning systems considered here is (classical) higher-order logic (HOL) $[22,11,3]{ }^{1}$ HOL is a typed logic which is built on top of the simply typed $\lambda$-calculus. The set of simple types $\mathcal{T}$ contains all types that are freely generated using the binary function type constructor $\rightarrow$ and a set of base types, including $o$ and $\iota$ for the type of Booleans and individuals, respectively. The terms of HOL are given by $(\tau, \nu \in \mathcal{T})$ :

$$
s, t::=c_{\tau}\left|X_{\tau}\right|\left(\lambda X_{\tau} . s_{\nu}\right)_{\tau \rightarrow \nu} \mid\left(s_{\tau \rightarrow \nu} t_{\tau}\right)_{\nu}
$$

where $c_{\tau} \in \Sigma_{\tau}$ is a constant symbol of type $\tau$ from the signature $\Sigma:=\bigcup_{\tau \in \mathcal{T}} \Sigma_{\tau}$ and $X_{\tau} \in \mathcal{V}$ is a variable from a set $\mathcal{V}$ of countably infinitely many variables for each type. Terms of the form $\left(\lambda X_{\tau} . s_{\nu}\right)_{\tau \rightarrow \nu}$ and $\left(s_{\tau \rightarrow \nu} t_{\tau}\right)_{\nu}$ are called abstractions and applications, respectively. By convention, applications are left-associative whereas the function type constructor $\rightarrow$ is rightassociative. We hence avoid unnecessary parentheses if possible. The type of a term is explicitly stated as subscript but may be dropped for legibility reasons if obvious from the context. Terms $s_{o}$ of type $o$ are called formulas.

In general, we require $\Sigma$ to contain a complete logical signature. To that end, we choose $\Sigma$ to consist of at least the primitive logical connectives for disjunction, negation, and, for each type, equality and universal quantification. ${ }^{2}$ Hence, we have $\left\{\bigvee_{o \rightarrow o \rightarrow o}, \neg_{o \rightarrow o},=_{\tau \rightarrow \tau \rightarrow o}^{\tau}, \forall_{(\tau \rightarrow o) \rightarrow o}^{\tau}\right\} \subseteq$ $\Sigma$ for all $\tau \in \mathcal{T}$. The remaining logical connectives can be defined as usual, e.g. conjunction by $\wedge_{o \rightarrow o \rightarrow o}:=\lambda s_{o} . \lambda t_{o} . \neg(\neg s \vee \neg t)$.

The semantics for HOL is meanwhile well-understood and can be found in the literature $[25,1,2,10]$ to which we refer for brevity. Validity of a formula $s_{o}$ is denoted $\models^{\mathrm{HOL}} s_{o}$.

For HOL, sophisticated automated and interactive theorem provers are available [7]. Prominent examples for the first kind of systems are Leo-II [13], Satallax [20] and TPS [4], and, for the latter kind, Isabelle/HOL [27].

\section{Quantified Multimodal Logic}

Quantified Multimodal Logic can be seen as an extension of classical higher-order logic as introduced in $\S 2$. To that end, the syntax of HOL is augmented with the modal operator $\square_{\boldsymbol{o} \rightarrow \boldsymbol{o}}$

\footnotetext{
${ }^{1}$ The brief introduction to HOL is mainly borrowed from [30] which, in turn, adapts the simplified notation of [26] for HOL.

${ }^{2}$ We will use infix notation for the usual binary logical connectives, i.e. write $(s \vee t)$ instead of $(\vee s) t$
} 


\begin{tabular}{c|c|c} 
Name & Axiom scheme & Frame condition \\
\hline $\mathrm{K}$ & $\square(\varphi \rightarrow \psi) \rightarrow(\square \varphi \rightarrow \square \psi)$ & - \\
$\mathrm{T}$ & $\square \varphi \rightarrow \varphi$ & reflexive \\
$\mathrm{B}$ & $\varphi \rightarrow \square \diamond \varphi$ & symmetric \\
$\mathrm{D}$ & $\square \varphi \rightarrow \diamond \varphi$ & serial \\
4 & $\square \varphi \rightarrow \square \square \varphi$ & transitive \\
5 & $\diamond \varphi \rightarrow \square \diamond \varphi$ & euclidean
\end{tabular}

Table 1: Axiom schemes for modal logics

(its dual, the $\diamond_{\boldsymbol{o} \rightarrow \boldsymbol{o}}$ operator, can be defined by $\diamond:=\lambda s_{\boldsymbol{o}} . \neg(\square \neg s)$ ). To distinguish between HOL and specialized logics in the remainder of this paper, we will give connectives and types in modal logics in boldface and the classical HOL connectives and types normal font.

Both modal operators can have varying interpretations (intended meaning) depending on the specific modal logic and application domain considered. The standard reading of $\square$ (resp. $\diamond$ ) is "necessarily" (resp. "possibly"). With this interpretation of the modal operators, the formula

$$
\square(\exists x \text {. big } x \wedge \diamond(\forall y \text {. smaller } y x))
$$

expresses that "necessarily there exists an $x$, that is big and possibly all $y$ are smaller than $x "$.

There exists a wide range of modal logics such as temporal logics, epistemic logics, deontic logics and conditional logics. All of these have a different interpretation of $\square$ and $\diamond$, which is mirrored by the modal operator's different axiomatizations. However, all modal logics share the common axiom $\mathrm{K}$

$$
\square(\varphi \rightarrow \psi) \rightarrow(\square \varphi \rightarrow \square \psi)
$$

and the necessitation principle (if $\varphi$ is valid, then so is $\square \varphi$ ).

Table 1 presents the most prominent of those axioms for specialized modal logics. As an example, consider the above mentioned deontic logic - a logic about obligations and permits - which includes the axiom scheme $\mathrm{D}$, reading as "if it is obligatory that $\varphi$ holds, then $\varphi$ is permitted". In more involved modal logics it may be necessary to introduce more than one $\square$ operator. For example, a common temporal logic, named Tense Logic, introduces two operators $\square^{f}$ and $\square^{p}$ (normally written as $\mathrm{G}$ and $\mathrm{H}$, respectively). The first one $\square^{f}$ reads as goes on, while the second one $\square^{p}$ reads as has been. In general, we can describe a multimodal logic by introducing a family of $\square^{i}$-operators, for $i \in I$, over an arbitrary index set $I$.

An appropriate semantics is obtained by adapting Henkin semantics for HOL to a Kripke semantics for these quantified multimodal logics (QMML). In this semantic a set of possible worlds is defined. Each formula is evaluated corresponding to a world. The modal operators change the reference of the currently evaluating world. Details on the semantics of QMML can be found elsewhere [12]. The validity of a QMML formula $s_{\boldsymbol{o}}$ is denoted $\models^{\mathrm{QMML}} s_{\boldsymbol{o}}{ }^{3}$.

Agent Knowledge Epistemic logic, the logic about knowledge, can be interpreted as a form of multimodal logic [24]. The $\square$-operators here represent "knowing" and are indexed with an agent's identifier from an index set $I$, referring to the particular agent whose knowledge is addressed. For example the sentence " $a$ knows", for an agent name $a \in I$, can be stated as $\square^{a}$.

\footnotetext{
${ }^{3}$ Dealing with a set of axioms $\mathcal{A}$ we are assuming global variant of the consequence relation $\mathcal{A} \models{ }^{\mathrm{QMML}} \boldsymbol{s}_{\boldsymbol{o}}$. In global consequence the axioms are assumed to hold everywhere (in every world). A more detailed explanation can be found in [32]
} 
While dealing with common knowledge scenarios, we often define an additional entity often called "fool" to state that every individual knows a fact (every fool knows). A common knowledge operator $C$ is often characterized by an infinite recursion

$$
C(\varphi)=\square^{\mathrm{fool}}(\varphi \wedge C(\varphi))
$$

A fact is common knowledge, if every body knows it, and everybody knows, that it is common knowledge. A more eligible version - dealing with a stronger version of the fool - can be stated with the following axioms.

$$
\begin{aligned}
\left(\square^{\text {fool }} \varphi\right) & \rightarrow \varphi \\
\left(\square^{\text {fool }} \varphi\right) & \rightarrow \square^{\text {fool }}\left(\square^{\text {fool }} \varphi\right) \\
\left(\square^{\text {fool }} \varphi\right) & \rightarrow \square^{a} \varphi \quad, \text { for all agents } a \in I
\end{aligned}
$$

The first axiom deals with the fact that every fool only knows facts that are indeed true. The second axiom captures the recursive definition of common knowledge by assuring the recursive expansion. The last axiom captures the distribution of common knowledge, i.e. stating that every agent in fact knows what is common knowledge.

Additionally epistemic logic introduces introspection for each agent: Adding $\square^{a} \varphi \rightarrow \square^{a} \square^{a} \varphi$ and $\neg \square^{a} \varphi \rightarrow \square^{a} \neg \square^{a} \varphi$ as an axiom scheme ensures that every agent knows whether or not he knows a fact. But for the purpose of the following puzzle we do not need either of these axioms.

\subsection{The Wise Men Puzzle}

A classical example dealing with knowledge between agents and implicit knowledge transfer is the wise men puzzle (also known in a variation as muddy forhead puzzle). It reads as follows:

Once upon a time, a king wanted to find the wisest out of his three wisest men. He arranged them in a circle and told them that he would put a white or a black spot on their foreheads and that one of the three spots would certainly be white. The three wise men could see and hear each other but, of course, they could not see their faces reflected anywhere. The king, then, asked to each of them to find out the color of his own spot. After a while, the wisest correctly answered that his spot was white. How could he know that?

In order to solve this problem in a formal logic setting, we have to give a logical representation of the natural language text. As the text deals with knowledge, we choose the epistemic multimodal logic as introduced above.

The first common information given to the wise men, is that one on them has a white spot, and there are, in fact, three of them.

$$
\begin{aligned}
& \square^{\text {fool }}(\text { white } a \vee \text { white } b \vee \text { white } c) \\
& \square^{\text {fool }}(a \neq b \wedge a \neq c \wedge b \neq c)
\end{aligned}
$$

The next information eligible to all wise men, is that two men can see the color of the third man. However, a priori there is no information available about one's own spot.

$$
\begin{array}{ll}
\square^{\text {fool }}\left(\text { white } x \rightarrow \square^{y}(\text { white } x)\right) & \text {, for all } x, y \text { with } x \neq y \\
\square^{\text {fool }}\left(\neg \text { white } x \rightarrow \square^{y}(\neg \text { white } x)\right) & \text {, for all } x, y \text { with } x \neq y
\end{array}
$$

Since we have only two possible colors for the spot, we model the color as a predicate white. The other color - black - is referred to by the negation of white. Finally, we encode the silence 
of the first two wise men by stating that "everybody knows, that $a$ (respectively $b$ ) does not know whether they have the white spot on the forehead".

$$
\begin{aligned}
& \square^{\text {fool }}\left(\neg \square^{a}(\text { white } a)\right) \\
& \square^{\text {fool }}\left(\neg \square^{b}(\text { white } b)\right)
\end{aligned}
$$

The puzzle's question can now be formulated as the proof problem that the last wise man can indeed deduce the color of his dot to be white.

$$
\square^{c}(\text { white } c)
$$

Using the above modal logic, the formalization of the puzzle is quite natural. The only extra information given by the formalization, is the consideration that identifies the silence of two wise men with them not knowing their dot's color. However, we claim that this is straight-forward and can be justified from the puzzle's description.

\section{Automation of QMML}

Automation of QMML is done using an indirection via HOL: The goal is hence to find equivalent formulations of QMML sentences in HOL. To that end, we first encode essential parts of the semantics of QMML within HOL, i.e. all logical ingredients such as connectives as well as the relevant meta-logical notions such as validity (in QMML, i.e. $\models^{\mathrm{QMML}}$ ). Subsequently, we formulate the original QMML problem using the above encoded notions and a translation scheme for constant symbols - which consists mostly of appropriate type-lifting. The procedure itself is described in more detail below (cf. §4.1). Finally, we can use ordinary theorem proving systems for HOL such as Isabelle/HOL for formulating the QMML problems by inputting the embedded variant of the original problem.

As a concrete example, the application of this process is depicted on the above presented wise-men puzzle using Isabelle/HOL in $§ 4.2$.

\subsection{Semantical Embedding}

For the particular instance of HOL used as target logic for the embedding, we assume the set of basic types to be $\{o, \iota, \mu\}$, where $o$ denotes the type of Booleans as before. Without loss of generality, $\iota$ is now identified with a (non-empty) set of worlds and the additional base type $\mu$ with a (non-empty) domain of individuals. ${ }^{4}$

QMML formulas are now identified with certain HOL terms (predicates) of type $\iota \rightarrow o$. They can be applied to terms of type $\iota$, which are assumed to denote possible worlds. The intuition here is that we evaluate the truth of a formula explicitly in a particular world. The definition of $\square$ and $\diamond$ are then defined as appropriate ${ }^{6}$ quantifications over the possible worlds. The type $\iota \rightarrow o$ is abbreviated as $\sigma$ ("type-lifted Booleans") in the remainder. Recall that the bold-face font denotes QMML terms and types, in order to distinguish them from those

\footnotetext{
4 The particular choice of whether $\mu$ or $\iota$ is identified with individuals, respectively worlds, is irrelevant and could be reversed. The selection here is motivated by the idea to stay as close as possible with the choices made in previous work. ${ }^{5}$

${ }^{5}$ In contrast to previous work where bold-face font was used for HOL, we use bold-face font for the embedded logic. This is motivated by the fact that the embedding in Isabelle/HOL also uses bold-face symbols for modal connectives.

${ }^{6}$ According to the Kripke-semantics interpretation of $\square s$, the embedding's denotation is achieved by checking whether $\left(\lceil s\rceil_{\iota \rightarrow o} v_{\iota}\right)$ holds for each accessible world $v_{\iota}$, where $\lceil s\rceil$ is the encoding of $s$ into HOL.
} 
of HOL. First, for each accessibility relation $r \in I$ of QMML, we introduce a constant symbol $r_{i \rightarrow i \rightarrow o}$ to the HOL signature. Then, for each type $\boldsymbol{\tau}$ of QMML we define the embedding (or type-lifting) $\lceil\boldsymbol{\tau}\rceil$ of $\boldsymbol{\tau}$ by

$$
\begin{aligned}
\lceil\boldsymbol{\mu}\rceil & =\mu \\
\lceil\boldsymbol{o}\rceil & =\sigma \\
\lceil\boldsymbol{\tau} \rightarrow \boldsymbol{\nu}\rceil & =\lceil\boldsymbol{\tau}\rceil \rightarrow\lceil\boldsymbol{\nu}\rceil
\end{aligned}
$$

We extend the definition of $\lceil$.$\rceil to QMML terms by$

$$
\begin{aligned}
\left\lceil\boldsymbol{c}_{\boldsymbol{\tau}}\right\rceil & =c_{\lceil\boldsymbol{\tau}\rceil} \\
\left\lceil\boldsymbol{X}_{\boldsymbol{\tau}}\right\rceil & =X_{\lceil\boldsymbol{\tau}\rceil} \\
\left\lceil\boldsymbol{\lambda} \boldsymbol{X}_{\boldsymbol{\tau}} . \boldsymbol{s}_{\boldsymbol{\nu}}\right\rceil & =\lambda\left\lceil\boldsymbol{X}_{\boldsymbol{\tau}}\right\rceil\left\lceil\boldsymbol{s}_{\boldsymbol{\nu}}\right\rceil \\
\left\lceil\boldsymbol{s}_{\boldsymbol{\tau} \rightarrow \boldsymbol{\nu}} \boldsymbol{t}_{\boldsymbol{\tau}}\right\rceil & =\left\lceil\boldsymbol{s}_{\boldsymbol{\tau} \rightarrow \boldsymbol{\nu}}\right\rceil\left\lceil\boldsymbol{t}_{\boldsymbol{\tau}}\right\rceil
\end{aligned}
$$

Hence, all the constant symbols and the variables are lifted to (non-bold) equivalents in HOL. Of course, the logical connectives of QMML lifted to HOL are here of particular interest, they are defined by:

$$
\begin{aligned}
\neg_{\sigma \rightarrow \sigma} & :=\lambda S_{\sigma} \cdot \lambda W_{\iota} \cdot \neg(S W) \\
\vee_{\sigma \rightarrow \sigma \rightarrow \sigma} & :=\lambda S_{\sigma} \cdot \lambda T_{\sigma} \cdot \lambda W_{\iota} .(S W) \vee(T W) \\
\Pi_{(\tau \rightarrow \sigma) \rightarrow \sigma}^{\tau} & :=\lambda P_{\tau \rightarrow \sigma} \cdot \lambda W_{\iota} \cdot \forall X_{\tau} . P X W \\
\square_{\sigma \rightarrow \sigma}^{r} & :=\lambda S_{\sigma} \cdot \lambda W_{\iota} \cdot \forall V_{\iota} . \neg(r W V) \vee S V
\end{aligned}
$$

Finally, we encode the notion of QMML validity by defining an appropriate abbreviation $\lfloor$.$\rfloor , grounding terms of type \sigma$ to type $o$ :

$$
\left\lfloor s_{\sigma}\right\rfloor:=\forall W_{i} . s W
$$

Theorem 1 (Soundness, Completeness). For all QMML formulas $\boldsymbol{s}_{\boldsymbol{o}}$ it holds that

$$
\models^{Q M M L} \boldsymbol{s}_{\boldsymbol{o}} \text { if and only if } \models^{H O L}\left\lfloor\left\lceil\boldsymbol{s}_{\boldsymbol{o}}\right\rceil\right\rfloor
$$

A proof is presented in earlier work [12].

The above presented embedding is not restricted to base modal logic K. In order to use stronger logics, we can easily add the frame conditions corresponding to the respective axiom scheme (cf. Table 1) as additional axioms. As an example, when modal logic KB is considered, we simply add the HOL axiom for symmetry, i.e. $\forall W_{\iota} \forall V_{\iota} . \neg(r W V) \vee r V W$, to the set of axioms. This is a major advantage over systems that have a hard-wired underlying logic.

Examples. The encoding is a straight-forward replacement of all modal logic symbols for their definiendum. The introductory example

$$
\square_{o \rightarrow o} \exists X_{\mu} \cdot \operatorname{big}_{\boldsymbol{\mu} \rightarrow \boldsymbol{o}} X \wedge \diamond_{\boldsymbol{o} \rightarrow \boldsymbol{o}} \forall Y_{\mu} \cdot \text { smaller }_{\boldsymbol{\mu} \rightarrow \boldsymbol{\mu} \rightarrow \boldsymbol{o}} Y X
$$

is hence mapped to the HOL proof problem

$$
\begin{aligned}
& \left\lfloor\left\lceil\square_{\boldsymbol{o} \rightarrow \boldsymbol{o}} \exists X_{\mu} \cdot \operatorname{big}_{\boldsymbol{\mu} \rightarrow \boldsymbol{o}} X \wedge \diamond_{\boldsymbol{o} \rightarrow \boldsymbol{o}} \forall Y_{\mu} \cdot \text { smaller }_{\boldsymbol{\mu} \rightarrow \boldsymbol{\mu} \rightarrow \boldsymbol{o}} Y X\right\rceil\right\rfloor \\
= & \left\lfloor\lambda U_{\iota} \cdot \forall W_{\iota} \cdot\left(U R W \rightarrow \exists X_{\mu} \cdot\left(\operatorname{big}_{\mu \rightarrow \sigma} X W \wedge \exists V_{\iota} \cdot\left(W R V \rightarrow \forall Y_{\mu} \cdot \text { smaller }_{\mu \rightarrow \mu \rightarrow \sigma} Y X V\right)\right)\right)\right\rfloor \\
= & \forall U_{\iota} \cdot \forall W_{\iota} \cdot\left(U R W \rightarrow \exists X_{\mu} .\left(\operatorname{big}_{\mu \rightarrow \sigma} X W \wedge \exists V_{\iota} .\left(W R V \rightarrow \forall Y_{\mu} \cdot \text { smaller }_{\mu \rightarrow \mu \rightarrow \sigma} Y X V\right)\right)\right)
\end{aligned}
$$


The structure of the formula is still the same, but we can see that the original formulas become predicates on worlds which are explicitly applied.

Concerning our wise men puzzle, e.g. the axiom

$$
\square_{o \rightarrow o}^{\text {fool }}\left(\text { white }_{\alpha \rightarrow o} a \rightarrow \square_{o \rightarrow o}^{b} \text { white }_{\alpha \rightarrow o} a\right)
$$

is analogously encoded as (let $\alpha=\iota \rightarrow \iota \rightarrow o$ for brevity)

$$
\forall W_{\iota} . \forall V_{\iota} . \text { fool }_{\alpha} W V \rightarrow\left(\text { white }_{\alpha \rightarrow \sigma} a V \rightarrow \forall U_{\iota} . b_{\alpha} V U \rightarrow \text { white }_{\alpha \rightarrow \sigma} a U\right) .
$$

As we can see, the type $\iota \rightarrow \iota \rightarrow o$ of the embedded modal operator's subscripts (the identifier of the agents) corresponds to the type of the accessibility relations, allowing to identify these two counterparts within the HOL encoding. It is important to note that expansions of the encoding can suitably be hidden from the user in modern proof assistant systems such as Isabelle/HOL. This will be illustrated below.

\subsection{Automation using Isabelle/HOL}

The practical employment of the above described semantical embedding for QMML in Isabelle/HOL is straight-forward and can be done in a separate theory file. This way, for a concrete application scenario, we can simply import the embedding without dealing with any technical details. The complete embedding of QMML is quite short (approx. 30 lines of code with line breaks) and is displayed in Fig. 1 (a).

The formalization of the wise men puzzle from $\S 3.1$ in Isabelle/HOL, as seen in Fig. 1 (b), imports the afore described QMML theory file (not shown in the figure) and states the respective axioms and proof problem. Note that the formalization within Isabelle/HOL differs only marginally from the original formulation. This is also due to the syntactically convenient definitions of the embedded modal operators which can simply be written down using Isabelle's GUI and abbreviation system. The internal tactics of Isabelle/HOL can easily prove the depicted conjecture within few milliseconds. The proof search can additionally by guided by Sledgehammer calls which transmit the proof problems to automated theorem provers. We therefore claim that the usability of our approach, especially when combined with a sophisticated tool like Isabelle/HOL, is indeed quite good.

\section{Summary}

In our tutorial we will show that the embedding of non-classical logics in HOL gives us an easy and fast way to implement specialized theorem provers. We have sketched this approach for quantified multimodal logics as a prominent example. However, this technique can be applied for a broad range of further non-classical logics, including conditional logics, description logics, hybrid logics, many-valued logics and many more.

Moreover, environments like Isabelle/HOL allow the user to input the non-classical formalizations quite comfortably. In the tutorial session we will give a more in-depth introduction into the development of a new embedding, understanding of given embeddings, and working with Isabelle/HOL to formalize own theories.

In the future, we are interested in further application areas to employ this kind of approach and handling of non-classical logics with pre-existing proving tools. In parallel we will embed further non-classical logics as well to establish a growing support for a great variety of relevant logics. 


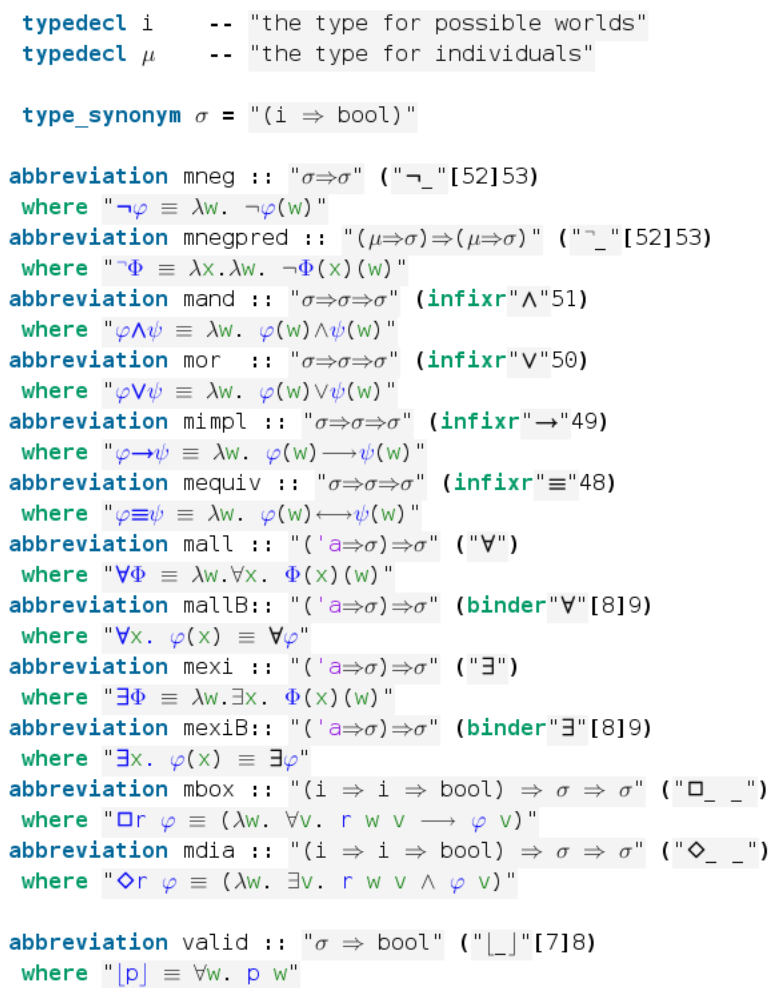

(a) Complete Embedding of QMML

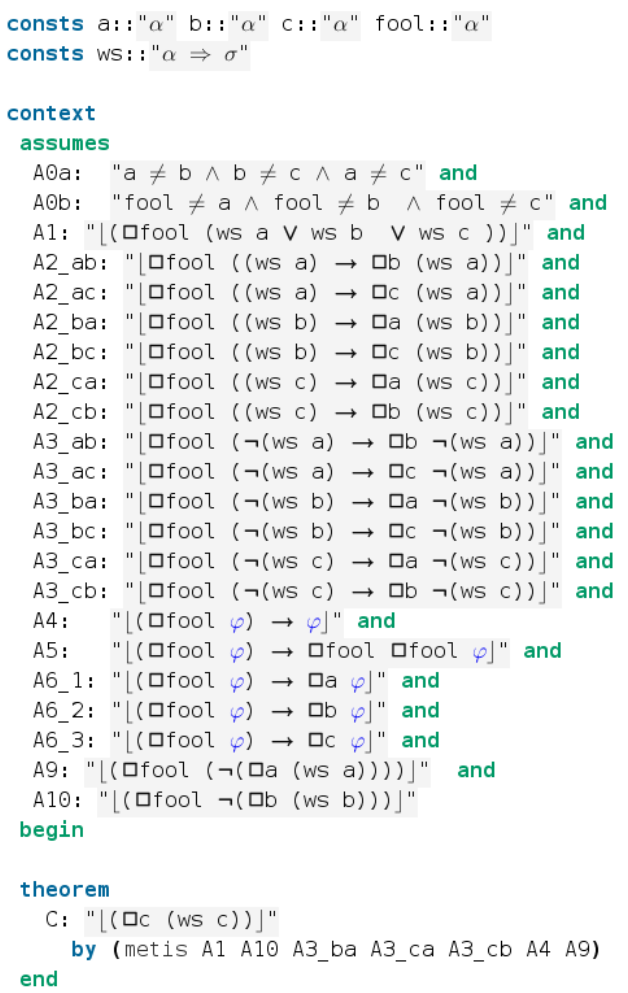

(b) Wise Men Puzzle

Figure 1: Application of the semantical embedding in Isabelle/HOL

Acknowledgements We thank Hans-Jörg Schurr and Tobias Gleißner for their comments and for proof reading this paper.

\section{References}

[1] P. B. Andrews. General models and extensionality. J. Symb. Log., 37(2):395-397, 1972.

[2] P. B. Andrews. General models, descriptions, and choice in type theory. J. Symb. Log., 37(2):385394, 1972.

[3] P. B. Andrews. Classical type theory. In John Alan Robinson and Andrei Voronkov, editors, Handbook of Automated Reasoning (in 2 volumes), pages 965-1007. Elsevier and MIT Press, 2001.

[4] P. B. Andrews, M. Bishop, S. Issar, D. Nesmith, F. Pfenning, and Hongwei Xi. TPS: A theoremproving system for classical type theory. J. Autom. Reasoning, 16(3):321-353, 1996.

[5] F. Baader, D. Calvanese, D. L. McGuinness, D. Nardi, and P. F. Patel-Schneider, editors. The Description Logic Handbook: Theory, Implementation, and Applications. Cambridge University Press, 2003.

[6] C. Benzmüller. Automating quantified conditional logics in HOL. In F. Rossi, editor, 23rd International Joint Conference on Artificial Intelligence (IJCAI-13), pages 746-753, Beijing, China, 2013. 
[7] C. Benzmüller. Higher-order automated theorem provers. In David Delahaye and Bruno Woltzenlogel Paleo, editors, All about Proofs, Proof for All, Mathematical Logic and Foundations, pages 171-214. College Publications, London, UK, 2015.

[8] C. Benzmüller. Invited talk: On a (quite) universal theorem proving approach and its application in metaphysics. In Hans De Nivelle, editor, TABLEAUX 2015, volume 9323 of LNAI, pages 213-220, Wroclaw, Poland, 2015. Springer. (Invited paper, mildly reviewed).

[9] C. Benzmüller. Cut-elimination for quantified conditional logic. Journal of Philosophical Logic, 2016.

[10] C. Benzmüller, C. Brown, and M. Kohlhase. Higher-order semantics and extensionality. Journal of Symbolic Logic, 69(4):1027-1088, 2004.

[11] C. Benzmüller and D. Miller. Automation of higher-order logic. In Dov M. Gabbay, Jörg H. Siekmann, and John Woods, editors, Handbook of the History of Logic, Volume 9 - Computational Logic, pages 215-254. North Holland, Elsevier, 2014.

[12] C. Benzmüller and L. Paulson. Quantified multimodal logics in simple type theory. Logica Universalis (Special Issue on Multimodal Logics), 7(1):7-20, 2013.

[13] C. Benzmüller, L. C. Paulson, N. Sultana, and F. Theiß. The higher-order prover LEO-II. Journal of Automated Reasoning, 55(4):389-404, 2015.

[14] C. Benzmüller and D. Scott. Automating free logic in Isabelle/HOL. In G.-M. Greuel, T. Koch, P. Paule, and A. Sommese, editors, Mathematical Software - ICMS 2016, 5th International Congress, Proceedings, volume 9725 of LNCS, pages 43-50, Berlin, Germany, 2016. Springer.

[15] C. Benzmüller and D. S. Scott. Axiomatizing category theory in free logic. http://arxiv.org/abs/1609.01493, 2016.

[16] C. Benzmüller, L. Weber, and B. Woltzenlogel-Paleo. Computer-assisted analysis of the AndersonHájek controversy. Logica Universalis, 2016. Accepted for publication; to appear in volume 10 (2016) issue 4 or volume 11 (2017) issue 1.

[17] C. Benzmüller and B. Woltzenlogel Paleo. Higher-order modal logics: Automation and applications. In Adrian Paschke and Wolfgang Faber, editors, Reasoning Web 2015, number 9203 in LNCS, pages 32-74, Berlin, Germany, 2015. Springer.

[18] C. Benzmüller and B. Woltzenlogel Paleo. The inconsistency in Gödels ontological argument: A success story for AI in metaphysics. In IJCAI 2016, 2016.

[19] P. Blackburn, J.F.A.K. van Benthem, and F. Wolter. Handbook of Modal Logic. Studies in Logic and Practical Reasoning. Elsevier Science, 2006.

[20] C.E. Brown. Satallax: An automated higher-order prover. In B. Gramlich, D. Miller, and U. Sattler, editors, Proc. of IJCAR 2012, volume 7364 of $L N A I$, pages 111 - 117. Springer, 2012.

[21] B. F. Chellas. Basic conditional logic. J. Philosophical Logic, 4(2):133-153, 1975.

[22] A. Church. A formulation of the simple theory of types. Journal of Symbolic Logic, 5:56-68, 1940.

[23] S. Gottwald. Many-valued logic. In Edward N. Zalta, editor, The Stanford Encyclopedia of Philosophy. Spring 2015 edition, 2015.

[24] V. Hendricks and J. Symons. Epistemic logic. In Edward N. Zalta, editor, The Stanford Encyclopedia of Philosophy. Fall 2015 edition, 2015.

[25] L. Henkin. Completeness in the theory of types. Journal Symbolic Logic, 15(2):81-91, 1950.

[26] R. Muskens. Higher order modal logic. Handbook of modal logic, 3, 2007.

[27] T. Nipkow, L.C. Paulson, and M. Wenzel. Isabelle/HOL: A Proof Assistant for Higher-Order Logic. Number 2283 in LNCS. Springer, 2002.

[28] R. C. Stalnaker. A theory of conditionals. In Ifs, pages 41-55. Springer, 1968.

[29] A. Steen and C. Benzmüller. Sweet SIXTEEN: Automation via embedding into classical higherorder logic. Logic and Logical Philosophy, 2016.

[30] A. Steen, M. Wisniewski, and C. Benzmüller. Agent-based HOL reasoning. In G.-M. Greuel, 
T. Koch, P. Paule, and A. Sommese, editors, Mathematical Software - ICMS 2016, 5th International Congress, Proceedings, volume 9725 of LNCS, Berlin, Germany, 2016. Springer. To appear.

[31] M. Wisniewski and A. Steen. Embedding of Quantified Higher-Order Nominal Modal Logic into Classical Higher-Order Logic. In Jens Otten Christoph Benzmller, editor, 1st International Workshop on Automated Reasoning in Quantified Non-Classical Logics (ARQNL 2014), Vienna, Austria, Proceedings, volume 33 of EasyChair Proceedings in Computing, pages 59-64. EasyChair, 2014.

[32] M. Wisniewski, A. Steen and C. Benzmüller. TPTP and Beyond: Representation of Quantified Non-Classical Logics. In Christoph Benzmller Jens Otten, editor, ARQNL 2016. Automated Reasoning in Quantified Non-Classical Logics, EPiC Series in Computing, EasyChair, 2016. To appear. 